\title{
Commencer en continuant : du rôle des constructions détachées en -ant en tête de chapitre
}

L'exemple d'Oudin, de Rosset et de Vaugelas

Starting by Continuing: The Role of -ant Detached Constructions at the Beginning of Chapters. The Example of Oudin, Rosset, and Vaugelas

\section{Mathieu Goux}

\section{OpenEdition}

\section{Journals}

Édition électronique

URL : http://journals.openedition.org/scolia/1362

DOI : $10.4000 /$ scolia.1362

ISSN : 2677-4224

Éditeur

Presses universitaires de Strasbourg

Édition imprimée

Date de publication : 10 juillet 2020

Pagination : 115-131

ISBN : 979-10-344-0067-6

ISSN : $1253-9708$

Référence électronique

Mathieu Goux, "Commencer en continuant : du rôle des constructions détachées en -ant en tête de chapitre », Scolia [En ligne], 34 | 2020, mis en ligne le 10 juillet 2020, consulté le 10 juillet 2020. URL: http://journals.openedition.org/scolia/1362 ; DOI : https://doi.org/10.4000/scolia.1362 


\title{
Commencer en continuant: du rôle des constructions détachées en -ant en tête de chapitre L'exemple d'Oudin, de Rosset et de Vaugelas
}

\author{
Mathieu Goux \\ Université de Caen, CRISCO-EA 4255 \\ mathieu.goux@unicaen.fr
}

\section{Introduction}

Les études se consacrant à la grammaire de texte, et notamment aux phénomènes relevant de la cohérence et de la cohésion, s'intéressent le plus souvent à des unités allant au-delà des frontières phrastiques contemporaines marquées par la ponctuation. Qu'il s'agisse de la période (Berrendonner et al., 2012; Goux, 2017a) ou de la séquence textuelle (Le Goffic, 2008), ces nouveaux cadres d'analyse, dans les bornes desquels est étudiée la dynamique informationnelle de l'énoncé et sa progression thématique, ainsi que les phénomènes de cohésion comme les anaphores, permettent de mieux saisir comment un texte est perçu comme texte sans se limiter aux seuls observables morphosyntaxiques.

Il faut noter néanmoins que cette démarche, bien que s'affranchissant d'une observation par trop étroite des différents faits de textualité, ne permet pas nécessairement de relier ces remarques textuelles avec «l'objet texte», qu'il soit linguistiquement, culturellement ou littérairement constitué, dans toute sa matérialité et dans son intégralité, surtout. On peut arguer que l'analyse de la dynamique informationnelle d'une suite de périodes ou de séquences textuelles, sur l'ensemble d'un chapitre d'un roman voire d'un roman entier, 
conduit à mettre en retrait les observables linguistiques au profit d'une analyse en appelant davantage à la (grammaire) stylistique, voire à la rhétorique. Effectivement, si de telles analyses existent (Barthes, 1966; Lorian, 1973; Larthomas, 1994; Lecointe, 1997), les études purement linguistiques semblent davantage ici un moyen qu'une fin, un argument supplémentaire dans une démonstration au propos assez éloigné des préoccupations textuelles en tant que telles ${ }^{1}$.

Notre article se propose pourtant de réunir ces champs disciplinaires, en choisissant un objet dont, certes, les bornes linguistiques capitalisent sur les analyses de la grammaire de texte, mais dont l'emplacement général au sein de l'économie d'une œuvre nous permet d'ouvrir nos remarques vers une dimension plus large. Nous analyserons effectivement des problématiques liées à l'économie de romans de la période classique et notamment leur agencement chapitré, en lien avec des phénomènes de dynamique informationnelle, de prédication seconde et de continuité référentielle.

La recherche des unités d'un texte, quel qu'il soit, est effectivement une étape déterminante quant à ce type d'études (Le Goffic, 2008: 329); mais les découpes qu'opèrent les chercheurs au sein d'un continuum sont généralement artificielles, quand bien même certains indices, telle la ponctuation ou le jeu des référents, permettraient d'orienter intuitivement l'organisation d'un texte en différents grands mouvements thématiques pour faciliter les analyses. Il est cependant possible de s'appuyer, pour cette découpe, sur des bornes qui ont été préétablies par les auteurs: dans la mesure où un texte est par essence clos (Arrivé, 1969: 5), à sa gauche par le début de l'écriture, à sa droite par sa fin, nous pouvons toujours prendre en compte les tout premiers mots, ou les tout derniers mots, d'un roman, d'un article de presse, d'un essai... pour faciliter cette opération de découpage. Il est, après tout, légitime de considérer qu'au commencement absolu d'un texte n'apparaissent que des référents «flambant neufs» (Kleiber, 1994:

1 Sur cette réflexion, on pourra notamment renvoyer à l'article, déjà ancien, de Michel Arrivé, "Postulats pour la description linguistique des textes littéraires", qui apparaît comme une sorte de manifeste conciliant littérature et linguistique, s'élevant sur les ruines d'une stylistique qui semble «à peu près morte» (Arrivé, 1969: 3). 
116), tandis qu'à sa fin absolue, nous ne trouverons là que leurs ultimes mentions ${ }^{2}$.

Il est cependant un cas particulier, que nous aimerions aborder ici: les bornes intermédiaires de textes narratifs longs, et notamment les découpes chapitrées qui scandent, en parallèle de la répartition en «livres» ou en "parties», la progression des péripéties. Cette découpe, qui permet au lecteur de séquencer sa lecture de moments d'importance en moments d'importance, est effectivement ambiguë, dans la mesure où bien que le début d'un chapitre instaure de nouveaux éléments de progression et de nouveaux référents, il construit également une continuité avec le chapitre précédent duquel il reprendra plusieurs éléments, notamment les protagonistes que nous suivons au long de ce type de texte. Il faut donc pour les auteurs, en quelque sorte, "commencer en continuant», soit réinstaller de nouveaux éléments de compréhension tout en aménageant la continuité antérieure.

Nous proposons d'explorer ces questions en analysant un phénomène au cœur de cette problématique, les constructions détachées $(\mathrm{CD})$ en -ant, soit les subordonnées ou constructions participiales régies par un participe présent ou un gérondif ${ }^{3}$ et auxquelles est associé un contrôleur, un GN ou un pronom, implicite ou explicite, qui détermine le référent accomplissant l'action décrite par le groupe verbal. Le rôle de ces constructions consiste précisément à séquencer la continuité textuelle en apportant un «déjà-là " à la prédication principale prise en charge dans la phrase graphique par un verbe au mode personnel (Combettes, 1998; 2003; 2005). Plus précisément, ces constructions en -ant agissent sur trois niveaux de continuité: d'une part, du point de vue de la cohésion textuelle, par l'intermédiaire dudit contrôleur, qui noue une relation anaphorique avec une autre expression dans le texte. Comme le souligne Combettes:

Une des caractéristiques fondamentales de la $\mathrm{CD}$ est non seulement de s'appliquer à un référent (il n'y a pas de $\mathrm{CD}$

2 Ceci, évidemment, en excluant les débuts de récit dits in medias res, à l'instar du célèbre incipit "La marquise sortit à 5 heures» attribué à Valéry et qui pose d'intéressants problèmes de référence. Voir Genette (1966) pour une analyse des «frontières du récit».

3 Nous rappelons qu'en français classique, la distinction participe présent/gérondif n'est pas encore parfaitement établie (Fournier, 2002: 292-296). 
«impersonnelles», par exemple), mais aussi d'imposer, pourrait-on dire, une coréférence entre ce sujet sous-jacent et un référent recouvert par une expression située en principe dans la phrase. (Combettes, 1998: 13)

d'autre part, du point de vue de la cohérence textuelle, la forme en -ant ayant une valeur de prédication seconde s'articulant de différentes façons avec une prédication principale et participant, dès lors, à la conduite du propos, en association avec la cohésion textuelle qu'elle élabore.

[Les formes en -ant] permettent de traduire un état du sujet concomitant à lévénement auquel renvoie la forme verbale conjuguée, peuvent, par leurs caractéristiques mêmes, évoluer vers la prédication seconde; elles supposent en effet la présence d'un sujet sous-jacent en coréférence avec le sujet principal et correspondent, en quelque sorte, à des propositions réduites, elliptiques. (Combettes, 2003: $\$ 12$ )

enfin, du point de vue de la connexité, que nous définirons à la suite de Neveu (2007: 166) comme une relation de cohésion opérant «au niveau transphrastique et au niveau de la structure séquentielle du texte ou du discours». Dans la mesure où notre problématique nous invite à analyser des constructions en -ant en tête de chapitre, il est possible de les considérer comme agissant au niveau de la connexité, quand bien même ne feraient-elles pas partie, strico sensu, de la famille des «connecteurs», que les grammaires associent généralement à des formes non-verbales ${ }^{4}$.

Nous étudierons l'emploi de ces formes en -ant au début des chapitres du Don Quichotte de Cervantès, traduit par César Oudin et François de Rosset (1614 et 1618), et de la Vie d'Alexandre de QuinteCurce, traduit par Vaugelas (1653). Ces textes narratifs, outre cette découpe chapitrée qui les caractérise, ont été traduits à une période où, précisément, ces structures en -ant sont sur le déclin (Fournier, 2002: 309-310). Particulièrement, la traduction de Vaugelas ayant été corrigée par les Académiciens quelque cinquante ans après sa rédaction (1709, édité et présenté par Ayres-Bennett \& Caron, 1996), leurs remarques nous permettront de lire le sentiment des doctes sur

4 À l'instar de la $G M F$, qui désigne sous le terme de «connecteurs» des groupes prépositionnels ou adverbiaux, ou encore des conjonctions (Riegel et al., 2014: 1043-1057). 
l'évolution de la langue à ce propos. Également, la comparaison de ces textes avec les versions originales nous permettra d'observer les structures, tant espagnoles que latines, que ces auteurs retranscrivent et d'améliorer notre compréhension des stratégies mises en œuvre à ces moments critiques de leurs traductions.

\section{Définition et analyse du corpus}

Pour notre étude, nous avons effectué un relevé exhaustif des participes en -ant que nous trouvions en tête des chapitres des romans sélectionnés. Nous avons distingué trois catégories de constructions participiales, chacune participant à notre problématique, mais dont les distinctions conduiront cependant notre démonstration:

(i) Les constructions participiales dont le contrôleur est un référent dont la source se situe dans le chapitre antécédent, sans reprise anaphorique ultérieure:

1) [...] il vint à l'appeler Dulcinée du Toboso, parce qu'elle étoit native du Toboso, nom, à son avis, musical et rare et bien significatif, comme l'étoient tous les autres qu'il avoit imposés à lui et à tout ce qui appartenoit.

Chapitre II - De la première sortie que fit l'ingénieux don Quichotte de son pays.

Ces préparatifs étant donc ainsi faits, il ne voulut pas différer plus longtemps à effectuer sa délibération [...] (Oudin, 1614, I.25)

(ii) Les constructions participiales dont le contrôleur est un référent à la fois présent dans le chapitre en cours, mais également dans le chapitre antécédent.

2) ...il [Alexandre] passa dans la Syrie, \& envoya Parmenion devant un en Damas, où étoit le trésor de Darius.

13. Mais Alexandre ayant appris en chemin qu'un des Satrapes du Roi l'avoit devancé, craignant d'estre attaqué avec le peu de gens qu'il avoit, il resolut de faire venir du renfort. (Vaugelas, 1653, III.13)

5 Les références des exemples, outre l'auteur et l'année d'édition, indiquent également le livre et le chapitre étudiés. 
(iii) Les constructions dont le contrôleur est un référent présent exclusivement dans le chapitre en cours.

3) La nièce et la gouvernante, qui étoient sans doute de fort bonnes filles, le mirent dans le lit, là où elles lui donnèrent à manger et le traitèrent aussi bien que possible.

Chapitre LXXIV - Comme quoi don Quichotte tomba malade, du testament qu'il fit, et de sa mort.

Comme les choses humaines, nétant point éternelles, vont toujours en déclinant depuis leurs principes jusquà ce quelles parviennent à leur dernière fin, et principalement la vie des hommes, et comme celle de don Quichotte niavoit pas ce privilège du Ciel d'être toujours au même état et dans sa course, sa fin arriva lorsqu'il y pensoit le moins. (Rosset, 1618, II.74)

Nous avons également analysé la position de ces constructions participiales, en opposant celles se trouvant en tête absolue de chapitre (4) de celles se situant dans une position intermédiaire, en apposition et généralement entre un GN faisant office de contrôleur et un prédicat principal, régi par un verbe à un mode personnel (5). Cette distinction nous a semblé importante dans la mesure où les phénomènes de cohésion textuelle sont tributaires de la linéarité et de l'autonomisation des constituants, particulièrement en ce qui concerne les phénomènes d'accessibilité référentielle (Kleiber, 1994: 116). Ainsi, les exemples tels (4) se prêtent davantage à une rupture thématique que les participiales apposées (5).

4) [...] l'armée n'est-elle pas heritiere de toutes ces grandes richesses qu'il a laissée?

7. Ayant dit cela, il passa au travers de la foule [...] (Vaugelas, 1653, X.7)

5) [...] dont la postérité aprés tant de siecles fleurit encore parmi ces Nations, à cause de la memoire d'Alexandre.

7. Mais le Roi des Scythes qui sont au-delà du Tanaïs, voyant que cette Ville bâtie sur ce fleuve, étoit un joug qu’on lui mettoit sur le col, envoya son frere nommé Carthasis [...] (Vaugelas, 1653, VII.7)

Ces précautions prises, nous avons ainsi relevé dix-huit occurrences dans le Quichotte pour trente dans le Quinte-Curce. Ce faible nombre d'occurrences nous a permis d'opérer une analyse exhaustive de 
celles-ci, et quand bien même nous ne prétendrions pas aboutir à une conclusion générale de cette question, que ce soit sur le genre du texte narratif ou sur la période classique, elle nous permet d'avoir une vision d'ensemble de cette problématique au sein de ces œuvres. Il faut néanmoins mesurer ce que dissimulent ces chiffres: effectivement, ces constructions détachées représentent près d'un sixième des incipits de chapitre du Quichotte (18/126, 14,2\%), et près d'un tiers des incipits de chapitre du Quinte-Curce (30/98, 30,6\%). Autrement dit, même si nous n'avons pas suffisamment d'occurrences pour mener une analyse quantitative fiable, l'importance des constructions détachées participiales à ce moment critique de la progression romanesque paraît notable dans ce corpus précis.

\section{Stratégies de la continuité}

Ces constructions détachées font pleinement partie des différentes stratégies exploitées par les auteurs pour gérer leur progression chapitrée, et on les trouve donc aux côtés d'autres outils introductifs, tels les infinitifs prépositionnels (6a), les subordonnées explicatives (6b), circonstancielles (6c), ou divers groupes prépositionnels relevant de la catégorie générale des connecteurs (6d). Ces différentes structures prennent chacune en charge la continuité narrative, en rappelant de façon plus ou moins elliptique une information antérieure, généralement concernant les faits et gestes d'un personnage. On notera cependant que ces éléments de continuité peuvent porter tant sur la diégèse, en explicitant la chronologie des actions accomplies (6b, 6c et $6 \mathrm{~d})$, que sur la narration elle-même, l'auteur signalant un retour à son propos premier après une digression plus ou moins importante (6a).

6a) Chapitre XXVI - Où se poursuivent les finesses d'amoureux que fit don Quichotte en la Sierra Morena.

Et, pour revenir au conte de ce que fit le chevalier de la TristeFigure, depuis qu'il se vit seul [...] (Oudin, 1614, I.26)

6b) Chapitre XVI - De ce qui advint à l'ingénieux chevalier en cette taverne qu'il s'imaginoit être un château.

Le tavernier, qui vit ainsi don Quichotte en travers sur l'âne, demanda à Sancho quel mal il avoit. (Oudin, 1614, I.16) 
6c) 5. Quand il eut traversé toute l'Hircanie, Artabaze, de qui nous avons parlé comme de l'homme du monde le plus fidele à Darius [...] (Vaugelas, 1653, VI.5)

6d) 1. En ce même tems, ou environ, Cleãdre, Eracon, Agachon, \& Sitalces, arriverent à la Cour [...] (Vaugelas, 1653, X.1)

Comme les formes en -ant opèrent sur trois plans d'organisation textuelle concomitamment, soit la cohérence textuelle, la cohésion et la connexité, elles demeurent des outils de choix pour les auteurs au regard de ces précédents exemples: les connecteurs (6d) n'agissent effectivement que du point de vue de la connexité en l'absence de référents ou de prédicats. Quant aux subordonnées, relatives (6b) ou circonstancielles (6c), la présence d'un référent explicite les empêche d'avoir la plasticité transphrastique des constructions en -ant. Enfin, les infinitifs (6a), bien que participant de la cohérence et de la connexité, ne prennent pas en charge la cohésion textuelle du fait de l'absence de référent sujet, explicite comme implicite.

Comme nous l'indiquions cependant en introduction, les constructions en -ant sont en diminution au long de la période classique. Les Académiciens fustigent notamment leur emploi trop soutenu chez Vaugelas: "Cette narration est trop remplie de participes entassés les vns sur les autres» (Ayres-Bennett \& Caron, 1996: 115 ), et Fournier (2002: 309-310) souligne leur remplacement par des subordonnées circonstancielles ou des relatives, pour pallier «l'encombrement» (sic) de la phrase. L'étude de notre corpus montre pourtant que ce remplacement ne parvient pas à retranscrire tous les effets de sens produits par ces constructions en -ant. Nous avons observé deux emplois principaux de celles-ci dans la gestion de la continuité textuelle: un emploi mettant davantage l'accent sur la continuité référentielle, un autre mettant davantage l'accent sur la continuité prédicative. Nous précisons que ces emplois, que nous détaillons ci-après, ne sont pas exclusifs: quelle que soit l'occurrence analysée, nous retrouverons toujours cette participation aux trois plans de la dynamique textuelle. Il nous a semblé néanmoins que certains paramètres, notamment positionnels, influençaient notablement l'interprétation de ces structures. 


\subsection{Continuité référentielle}

La continuité référentielle que permet l'emploi de ces structures en -ant semble plus prononcée dans le cas où, d'une part, elles se situent en tête du chapitre, d'autre part, quand leur contrôleur n'est point présent dans le chapitre qu'elles introduisent. Ces cas de figure sont assez bien répartis dans notre corpus, comme en témoignent les relevés suivants.

\begin{tabular}{|l|c|c|}
\hline & Quichotte & Quinte-Curce \\
\hline CD en tête de chapitre & 7 (sur $18,39 \%)$ & 9 (sur 30,30\%) \\
\hline Contrôleur absent du chapitre & 9 (sur $18,50 \%)$ & 9 (sur 30,30\%) \\
\hline
\end{tabular}

Position de la $C D$ en tête de chapitre et absence du contrôleur dans le chapitre considéré

Lorsque le contrôleur renvoie à un référent absent du chapitre courant, ce qui s'opère souvent par le biais d'une anaphore conceptuelle (1 et 7), la $\mathrm{CD}$ a un rôle conclusif, puisque présentant l'ultime mention d'un maillon d'une chaîne de référence. Elle évite alors une rupture thématique trop forte entre les chapitres successifs, l'anaphore conceptuelle donnant l'impression «d'adoucir» la progression ${ }^{6}$.

6) [...] Don Quichotte fut grandement confus du conte d'André, et il fallut que tous se gardassent bien de rire pour ne le pas mettre tout à fait en colère.

Chapitre XXXII - Qui traite de ce qui arriva en l'auberge à toute la quadrille de don Quichotte.

Le bon dîner étant achevé, on sella incontinent, et, sans qu'il leur arrivât chose digne de se raconter, ils arrivèrent le lendemain à l'auberge, épouvantail et frayeur de Sancho Pança; et, encore qu'il ê̂t voulu n'y point entrer, il ne s'en put exempter. (Oudin, 1614, I.32)

La position initiale, quant à elle, introduit un cadre de discours, selon la définition de Charolles (1994: 128), soit un univers d'interprétation de l'énoncé subséquent dans lequel prendront place les futurs référents (8).

7) [...] Mais ce qui plus le fatiguoit étoit de ne se voir point armé chevalier, parce qu'il lui sembloit qu'il ne se pouvoit

6 Nous renvoyons aux analyses de Lecointe (1997:14), pour qui ces formes en -ant témoignent d'une «souplesse d'enchaînement», et construisent un style «élégant et naturel». 
légitimement mettre à aucune entreprise sans avoir reçu l'ordre de chevalerie.

Chapitre III - De la plaisante manière que tint don Quichotte pour se faire armer chevalier.

Étant ainsi travaillé de cette pensée, il abrégea son maigre souper de taverne, lequel étant achevé, il appela l'hôte, et, sétant enfermé avec lui en lécurie, il se mit à genoux devant lui, disant: [...] (Oudin, 1614, I.3)

Il est dès lors notable de voir l'association de ces deux paramètres au sein de notre corpus (7), association qui semble contre-intuitive, puisque la structure en -ant se chargerait à la fois d'être la dernière manifestation d'un référent spécifique, tout en délimitant l'univers d'interprétation de celui-ci. C'est qu'en vérité, et à l'encontre de ce qui s'observe traditionnellement avec ces structures cadratives (Charolles, op. cit.; Combettes, 2005: 38-40), cet univers ne s'étend point «vers la droite» et le chapitre courant, mais bien vers la gauche, vers le chapitre antécédent. Ce geste englobant, participant de la cohésion, de la cohérence et de la connexité, aménage la transition de chapitre en chapitre en se superposant à la découpe générale opérée par le texte, ce qui crée une continuité sensible dans la lecture. Pour ainsi dire, nous aurions là une sorte de rappel narratif, un "précédemment dans le roman» entièrement englobé dans son économie. Ces structures indiquent qu'il n'y a aucune ellipse entre les deux chapitres subséquents et qu'ils doivent se saisir dans une continuité temporelle immédiate.

Nous retrouvons ce rôle de marqueur de continuité référentielle, agissant entre deux chapitres successifs, dans un certain nombre d'occurrences mettant en jeu un contrôleur présent et dans le chapitre antécédent, et dans le chapitre courant: les auteurs emploient parfois un autre rappel anaphorique leur permettant d'assurer la continuité de façon prospective, là où la $\mathrm{CD}$ se charge de la continuité rétrospective. Nous avions cela dans l'exemple (2).

2) [...] il [Alexandre] passa dans la Syrie, \& envoya Parmenion devant un en Damas, où étoit le trésor de Darius.

13. Mais Alexandre ayant appris en chemin qu'un des Satrapes du Roi l'avoit devancé, craignant d'estre attaqué avec le peu de gens qu'il avoit, il resolut de faire venir du renfort. (Vaugelas, 1653, III.13) 
Cette occurrence est commentée par l'Académie française (AyresBennett \& Caron, 1996: 90), mais cette dernière ne met en avant que le besoin de coordonner les deux participes présents ayant appris... et craignant..., sans doute pour faciliter l'opération de repérage du contrôleur du second. Le pronom il en prolepse, quant à lui, assure la continuité référentielle du chapitre courant, là où Alexandre, véritable contrôleur des formes en -ant, participe du lien avec le chapitre antécédent.

\subsection{Continuité prédicative}

Dans le cas où le contrôleur de la $\mathrm{CD}$ est un référent «flambant neuf», chose assez rare ${ }^{7}$, le rôle de celle-ci se départ notablement du cas précédent. Il s'agit ici moins d'assurer une continuité référentielle qu'une continuité d'ordre prédicatif, liée à la façon dont les différents événements se succèdent chronologiquement. La CD permet dès lors d'opérer une transition événementielle, en clôturant ce qui était initialement, à la fin du chapitre antécédent, un événement de premier plan, pris en charge par des modes verbaux personnels, avant l'établissement d'une nouvelle prédication principale (9)8.

8) Le Roi se ressouvenant du déplaisir qu'il avoit eu d'avoir tué Clitus, à cause de l'indiscretion de sa langue, retint sa colere, mais ne peut s'empescher de dire, que l'envie étoit elle-même son bourreau.

13. Le lendemain, les Ambassadeurs d'Abisares étans venus trouver le Roi, lui remirent suivant leur pouvoir, tous les États de leur Maître [...] (Vaugelas, 1653, VIII.13)

Cette transition événementielle a dès lors un rôle conclusif, si ce n'est qu'elle agit à un autre niveau de compréhension textuelle, liée moins à l'identification des différents acteurs de la prédication qu'à la prédication elle-même, en intercalant entre deux événements d'importance déterminante dans la compréhension, prise en charge par les modes verbaux personnels, un événement secondaire, pris

7 Nous n'avons trouvé cela que dans deux occurrences du Quichotte, et une seule dans Quinte-Curce.

8 "[Les formes en -ant] équival[ent] à une proposition dont l'action tend à égaler en importance celle du verbe principal» (Skerlj, cité par Lecointe, 1997: 10). Nous renvoyons également à Combettes $(2003: \$ 12)$, cité plus haut. 
en charge par la structure en -ant. Nous serions dans une sorte de zone mitoyenne entre le palier de la cohésion textuelle, fondée sur l'identification d'éléments identiques d'unité textuelle à unité textuelle, et le palier de la cohérence textuelle, fondée sur la dynamique informationnelle et sur la succession prédicative. Nous pouvons rapprocher ces occurrences de celles mettant en jeu la conjonction (ce)pendant que (10 et 11), dont l'origine étymologique, puisqu'issue du participe présent du verbe pendre, participe à notre problématique.

9) [...] Et enfin quel chevalier errant y a-t-il eu, $y$ a-t-il ou $y$ aura-t-il au monde qui n'ait le courage de donner lui seul quatre cents coups de bâton à quatre cents archers qui se trouveront devant lui?»

Chapitre XLVI - De la notable aventure des archers et de la grande fierté de notre bon chevalier don Quichotte.

Cependant que don Quichotte disoit ceci, le curé persuadoit aux archers que don Quichotte étoit hors de son bon sens [...] (Oudin, 1614, I. 46)

10) [...] puisque c'est vous que nous avez mis en butte à sa colere.

2. Pendant qu'Amyntas poursuivoit ainsi, ceux qui avoient été envoyez aprés son frere Polemon, qui sen étoit fuï, arriverent \& le ramenerent lié. (Vaugelas, 1653, VII.2)

On notera que dans ces deux derniers exemples, et à l'instar de ce que nous avons observé dans la sous partie 2.1., la reprise d'un référent antécédent, don Quichotte et Amyntas respectivement, comme sujet de ces subordonnées circonstancielles, crée une continuité avec le chapitre précédent, qui vient s'ajouter à la continuité prédicative. Le rôle général de ces trois derniers exemples nous semble cependant identique: ces structures hiérarchisent les différents événements et créent une zone tampon balisant la succession de deux prédicats principaux, situés dans deux chapitres distincts. Cela permet de les interpréter comme se succédant dans un délai temporel proche et de compenser la segmentation opérée par la découpe chapitrée en assurant la continuité du propos.

\section{Influences de la traduction}

Les correspondances entre les textes français et le texte espagnol et latin laissent apparaître des caractéristiques qui méritent une sous- 
partie dédiée. Quelle que soit la langue source considérée, ces différents systèmes linguistiques ont des structures proches des CD en -ant, dans la mesure où elles mettent à profit des modes non-personnels que l'on traduit généralement en français par un participe présent. Ainsi, en espagnol (12), le gérondif (gerundio) peut être traduit directement en français par un participe présent, tandis qu'en latin (13), le participe présent restitue généralement un participe employé dans le cadre d'un ablatif absolu. Ces stratégies de traduction sont, de loin, les plus nombreuses dans notre corpus ${ }^{9}$, et on trouve donc régulièrement une correspondance directe entre les formes linguistiques, le verbe participial latin ou espagnol étant directement traduit par un participe présent français, malgré les écarts qui peuvent toucher la traduction des autres éléments des propositions concernées.

11) Capitulo V-Donde se prosigue la narracion de la desgracia de nuesto caballero

Viendo, pues, que, en efeto, no podía menearse, acordó de acogerse a su ordinario remedio [...] (Cervantès, 1605, I.5)

Chapitre V-Suite de la disgrâce de notre chevalier.

Voyant donc quen effet il ne se pouvoit remuer, il savisa d'avoir recours à son remède ordinaire [...] (Oudin, 1614, I.5)

12) XIII. Alexander, audito Darium mouisse ab Ecbatanis, omisso itinere quod patebat in Mediam [...] (Quinte-Curce, v. I ${ }^{\text {er }}$ s., V.13)

13. Alexandre ayant sçu que Darius étoit parti d'Egbarane, laissa le chemin de la Medie [...] (Vaugelas, 1653, V.13)

Aux côtés de ces traductions plus ou moins littérales cependant, Oudin, Rosset et Vaugelas ont choisi de restituer par des subordonnées en -ant d'autres types de construction. Ainsi Rosset a-t-il traduit en (14) un pretérito imperfecto (oyera) par un participe présent (lisant), ou encore le verbe au subjonctif d'une subordonnée circonstancielle introduite par Como (15) ou un infinitif $(16)^{10}$, tandis que Vaugelas choisit généralement de restituer par un participe présent ce qui était,

911 occurrences sur 18 dans le Quichotte (61\%) et 17 sur 30 (57\%) dans le QuinteCurce.

10 On remarquera, sur cette question, qu'Oudin est bien plus fidèle au texte original que Rosset, ce qui marque la différence de sensibilité entre les deux traducteurs. 
chez Quinte-Curce, un verbe à un mode personnel, parfois même au sein d'une proposition indépendante (17).

13) Capitulo XLIII - De los consejos segundos que dio don Quijote a Sancho Panza

¿QUIÉN oyera el pasado razonamiento de don Quijote que no le tuviera por persona muy cuerda y mejor intencionada? (Cervantès, 1615, II.43)

Chapitre XLIII - Des seconds conseils que donna don Quichotte à Sancho Pança.

Qui est-ce qui, lisant le discours précédent de don Quichotte, ne l'estimeroit point une personne fort sage et encore mieux portée de bonne volonté? (Rosset, 1618, II.43)

14) Capitulo LXXIV - De como don Quijote cayo malo, y del testamento que hizo, y su muerte

COMO las cosas humanas no sean eternas, yendo siempre en declinación de sus principios hasta llegar a su último fin [...] (Cervantès, 1615, II.74)

Chapitre LXXIV - Comme quoi don Quichotte tomba malade, du testament qu'il fit, et de sa mort.

Comme les choses humaines, neetant point éternelles, vont toujours en déclinant depuis leurs principes jusquà ce quelles parviennent à leur dernière fin [...] (Rosset, 1618, II.74)

15) Capitulo LXVI - Que trata de lo que vera el que lo leyer, o lo oira el que lo escuchare leer

AL SALIR de Barcelona, volvió don Quijote a mirar el sitio donde había caído, y dijo [...] (Cervantès, 1615, II.56)

Chapitre LXVI - Qui traite de ce que verra celui qui le lira, ou entendra celui qui lécoutera lire.

Don Quichotte, en sortant de Barcelone, se retourna pour regarder le lieu de sa chute, et proféra ces paroles [...] (Rosset, 1618, II.56)

16) XIII. Postero die legati Abisarae adiere regem. Omnia dicioni eius, ita ut mandatum erat, permittebant [...] (Quinte-Curce, v. ${ }^{\text {er }}$ s., VIII.13)

13. Le lendemain, les Ambassadeurs d'Abisares étans venus trouver le Roi, lui remirent suivant leur pouvoir, tous les États de leur Maître [...] (Vaugelas, 1653, VIII.13) 
Si ces variantes se retrouvent souvent en équivalence avec les structures en $-a n t^{11}$ dans les travaux de traduction compte tenu de leur spécificité en langue française, on appréciera cependant leur emploi dans ces œuvres. Nous considérons notamment qu'au regard des verbes personnels qu'elles restituent, elles balisent la dynamique informationnelle du texte narratif dans ces transitions chapitrées en proposant des arrière-plans événementiels aux actions principales. Quand bien même un grand nombre de nos occurrences seraient issues d'une traduction littérale de structures équivalentes dans les langues sources, ces variantes montrent que les auteurs emploient les constructions en -ant à dessein pour faciliter la progression, tant référentielle qu'événementielle, de leurs romans. Cela permet de considérer le texte comme un continuum dont les frontières véritables demeurent le début du tout premier chapitre et la fin de l'ultime, les découpes intermédiaires devant s'entendre moins comme de nouveaux commencements que comme une forme artificielle de segmentation, que les structures en -ant transcendent pour élaborer un continuum textuel ininterrompu.

\section{Conclusion}

Ce parcours des constructions en -ant inaugurant certains chapitres du Quichotte et de la Vie d'Alexandre a permis de mettre en lumière leur rôle au sein de la progression de l'énoncé. À la fois outils de cohérence, de cohésion et de connexité textuelles, elles permettent d'amoindrir la rupture que provoque l'irruption d'un nouveau chapitre dans les romans en proposant une continuité, tant référentielle que prédicative, entre le propos antécédent et le propos subséquent.

Leur diminution fréquentielle à l'époque classique cependant, et leur remplacement par des structures approchantes, a conduit les auteurs à mettre de côté cet outil d'une grande plasticité et à envisager de nouveaux outils de progression textuelle. Partant, même si les problématiques d'écriture, c'est-à-dire ce besoin de "commencer en continuant», demeurèrent les mêmes, cela n'a pu qu'aller avec une

11 Pour la langue latine et particulièrement le Quinte-Curce de Vaugelas, on se reportera à Goux (2017b). Pour la langue espagnole, on consultera Hernández (2010), et particulièrement les pages 100-104. 
segmentation plus forte opérée par les chapitres, et une nouvelle appréhension du genre du texte narratif long.

\section{Bibliographie}

ARRIVÉ M. (1969), Postulats pour la description linguistique des textes littéraires, Langue française 3, 3-13.

AYRES-BENNETT W. \& CARON P. (1996), Les remarques de l'Académie française sur le Quinte-Curce de Vaugelas, Paris, Presses de l'École Normale Supérieure.

BARTHES R. (1966), Introduction à l'analyse structurale des récits, Communications 8, 1-27.

BERRENDONNER A. et al. (2012), Grammaire de la période, Berne, Peter Lang.

CERVANTĖS M. de, [1605, 1615] (1866), El ingenioso hidalgo Don Quijote de la Mancha, Amsterdam, F. A. Brockhaus.

CHAROLLES M. (1994), Cohésion, cohérence et pertinence du discours, Travaux de linguistique 29, 125-151.

COMBETTES B. (1998), Les constructions détachées en français, Paris, Ophrys.

COMBETTES B. (2003), Aspects diachroniques des constructions à détachement, Cahiers de praxématique 40, article 3.

COMBETTES B. (2005), Les constructions détachées comme cadres de discours, Langue française 148, 31-44.

FOURNIER N. (2002), Grammaire du français classique, Paris, Belin.

GENETTE G. (1966), Frontières du récit, Communications 8, 152-163.

GOUX M. (2017a), De la période à la phrase: le témoin lequel, in Prévost S. \& Fagard B. (éds), Le français en diachronie: dépendances syntaxiques, morphosyntaxe verbale, grammaticalisation, Berne, Peter Lang, 133-154.

GOUX M. (2017b), Le pronom-déterminant lequel dans le QuinteCurce de Vaugelas, in Ponchon T. et al. (éds), Mots de liaison et d'intégration, Amsterdam, John Benjamins, 135-146.

HERNANDEZ D.G. (2010), La traduction français-espagnol des constructions absolues dans la presse, Synergies Espagne 3, 95-106.

KLEIBER G. (1994), Anaphores et pronoms, Louvain-la-Neuve, Duculot.

LARTHOMAS P. (1994), Théories linguistiques de l'école romantique: le cas de Victor Hugo, Romantisme 86, 67-72. 
LE GOFFIC P. (2008), Phrase, séquence, période, in Van Raemdonck D. (éd.), Modèles syntaxiques. La syntaxe à l'aube $d u x_{x I}{ }^{e}$ siècle, Berne, Peter Lang, 329-356.

LECOINTE J. (1997), Le style en -ant au Xvi siècle en France: conscience syntaxique et options stylistiques, L'information grammaticale $75,10-14$.

LORIAN A. (1973), Tendances stylistiques dans la prose narrative française $d u X I^{e}$ siècle, Paris, Klincksieck.

NEVEU F. (2007), Détachement et connexité, in Guimier C. (éd.), Éléments de relation: de la phrase au texte, Caen, Presses Universitaires de Caen, 165-177.

OUDIN C. \& ROSSET F. de, [1614, 1618] (1884), L'Histoire de Don Quichotte de la Manche, Paris, Jouaust.

QUINTE-CURCE [v. I ${ }^{\text {er }}$ s.] (1709), Historiarum Alexandri Magni Libri, in Vaugelas, C. F. de, [1653] (1709).

RIEGEL M. et al. (2014), Grammaire méthode du français, 5éd., Paris, Presses Universitaires de France.

VAUGELAS C. F. de, [1653] (1709), Quinte-Curce, De la Vie et des actions d'Alexandre le Grand, Paris, Brunet. 